\title{
Hyperelliptic components of the moduli spaces of quadratic differentials with prescribed singularities
}

Erwan Lanneau

\begin{abstract}
Moduli spaces of quadratic differentials with prescribed singularities are not necessarily connected. We describe here all cases when they have a special hyperelliptic connected component.

We announce the general classification theorem: up to the four exceptional cases in low dimensional stratum, any stratum of meromorphic quadratic differentials is either connected, or has exactly two connected components. In this last case, one component is hyperelliptic, the other not.
\end{abstract}

Mathematics Subject Classification (2000). 32G15, 30F30, 30F60, 58F18.

Keywords. Quadratic differentials, moduli space, measured foliations, Teichmüller geodesic flow.

\section{Introduction}

A meromorphic quadratic differential $\psi$ on a Riemann surface $M_{g}^{2}$ of genus $g$ is locally defined by $\psi=f(z)(d z)^{2}$ where $f(z)$ is a meromorphic function defined on a chart $(U, z)$. If we have the form $\psi=g(w)(d w)^{2}$ where $g(w)$ is a meromorphic function on $V$, the transition functions on $U \cap V$ satisfies

$$
\frac{f(z)}{g(w)}=\left(\frac{d w}{d z}\right)^{2} \text {. }
$$

In this paper we consider quadratic differentials having only simple poles, if any. We denote by $\mathcal{Q}_{g}$ the moduli spaces of pairs $\left(M_{g}^{2}, \psi\right)$, where $M_{g}^{2}$ is a Riemann surface and $\psi$ a meromorphic quadratic differential on it. Note that we have divided by the mapping class group $\operatorname{Mod}(g)$.

Let $\left(k_{1}, \ldots, k_{n}\right)$ be the orders of singularities of $\psi$, where $k_{i}>0$ corresponds to a zero $z^{k_{i}}(d z)^{2}$ of order $k_{i}$ and $k_{j}=-1$ corresponds to a simple pole $\frac{1}{z}(d z)^{2}$ of a quadratic differential. It is a classical result following of the Gauss- $\stackrel{z}{z}$ onnet formula that $\sum k_{i}=4 g-4$. 
Locally, in a simply-connected neighborhood of a nonsingular point, a quadratic differential can be presented as a square of an Abelian differential, but globally it is not the case in general. In this paper we consider only those quadratic differentials which are not globally the squares of Abelian differentials.

The moduli space $\mathcal{Q}_{g}$ is naturally stratified by the types of the singularities. We denote by $\mathcal{Q}\left(k_{1}, \ldots, k_{n}\right) \subseteq \mathcal{Q}_{g}$ the stratum of quadratic differentials $\left[M_{g}^{2}, \psi\right] \in \mathcal{Q}_{g}$ which are not the global squares of Abelian differentials, and which have the singularity pattern $\left(k_{1}, \ldots, k_{n}\right)$, where $k_{i}$ takes values in $\{-1,0,1,2, \ldots\}$. In some situations we consider the strata of pairs $\left[\psi, M_{g}^{2}\right]$ where the Riemann surface is provided with some fixed number of marked points, which are nonsingular points of the quadratic differential $\psi$. By convention we let $k_{i}=0$ when the point $P_{i}$ is, actually, just a marked point. Sometimes we shall call the marked points the fake zeros, and the zeros $P_{j}$ with $k_{j}>0$ the true zeros. We use the exponential notation $k^{m}$ for $k, k, k, \ldots, k$ repeated $m$ times. For example, $\mathcal{Q}\left(1^{4}, 8,2,3^{2}\right)$ stands for $\mathcal{Q}(1,1,1,1,8,2,3,3)$.

We are interested in the description of connected components of the moduli spaces $\mathcal{Q}\left(k_{1}, \ldots, k_{n}\right)$. In this paper we describe all connected components of a special type: the hyperelliptic ones. A complete description of all connected components of the moduli spaces $\mathcal{Q}\left(k_{1}, \ldots, k_{n}\right)$ can be found in [La2]. It will be the subject of a forthcoming paper.

Remark 1. In [KoZo] Kontsevich and Zorich classified connected components of the moduli spaces of Abelian differentials, which implies the classification of the moduli spaces of those holomorphic quadratic differentials which are globally the squares of Abelian differentials.

Remark 2. In [Ma] and [Ve1], Masur and Veech have independently proved that the Teichmüller geodesic flow acts ergodically on each connected component of each stratum of the moduli space of quadratic differentials; the corresponding invariant measure being a finite Lebesque equivalent measure.

Motivated by this result, the classification of connected components of the strata coincide with the classification of ergodic components of the Teichmüller geodesic flow.

The paper has the following structure. In section 2 we present an overview of the basic properties of quadratic differentials. In section 3 we construct the hyperelliptic connected components and prove Theorem 1, that is our list is complete. In section 4 we present the well-known relation between quadratic differentials and measured foliations. Then we use measured foliations to prove Theorem 2: strata with a hyperelliptic component are not connected except some particular cases in low genera. We draw from our two main results the following 
Corollary 1. - The three following series of strata in $\mathcal{Q}_{g}$

$$
\begin{array}{l|r}
\mathcal{F}_{2}=\{\mathcal{Q}(4(g-k)-6 ; 4 k+2) & 0 \leq k \leq g-2\} \\
\mathcal{F}_{3}=\{\mathcal{Q}(4(g-k)-6 ; 2 k+1 ; 2 k+1) & 0 \leq k \leq g-1\} \\
\mathcal{F}_{4}=\{\mathcal{Q}((2(g-k)-3 ; 2(g-k)-3 ; 2 k+1 ; 2 k+1) & -1 \leq k \leq g-2\}
\end{array}
$$

in genera $g \geq 3$ are non-connected. Each above stratum possesses one component which is hyperelliptic and at least one other which is not.

- The following strata corresponding respectively to genus 1 and 2 are connected and the whole stratum coincides with its hyperelliptic connected component

$$
\left\{\begin{array} { l } 
{ \mathcal { Q } ( - 1 , - 1 , 2 ) } \\
{ \mathcal { Q } ( - 1 , - 1 , 1 , 1 ) }
\end{array} \quad \text { and } \left\{\begin{array}{l}
\mathcal{Q}(2,2) \\
\mathcal{Q}(1,1,2) \\
\mathcal{Q}(1,1,1,1)
\end{array}\right.\right.
$$

In section 5 we announce the general classification theorem: up to four exceptional cases in low genera the strata of meromorphic quadratic differentials are either connected, or have exactly two connected components, and one of the two components is hyperelliptic.

\section{Mappings of the moduli spaces induced by ramified coverings of a fixed combinatorial type}

In this section we present some general information concerning the moduli spaces of quadratic differentials. All proofs and details can be found in papers [Ma], [Ve1], [MaSm], [Ve2], [Ko], [KoZo].

Theorem (Masur and Smillie). Consider a vector $\left(k_{1}, \ldots, k_{n}\right)$ with all $k_{i} \in \mathbb{N} \cup$ $\{-1\}$. Suppose that $\sum k_{i}=0 \bmod 4$ and $\sum k_{i} \geq-4$. Then the corresponding stratum $\mathcal{Q}\left(k_{1}, \ldots, k_{n}\right)$ is non-empty with the following four exceptions

$$
\mathcal{Q}(\emptyset), \mathcal{Q}(1,-1) \text { (in genus } g=1 \text { ) and } \mathcal{Q}(4), \mathcal{Q}(1,3) \text { (in genus } g=2 \text { ). }
$$

Remark 3. It is clear that if a quadratic differential has a singularity of odd order ( -1 for a pole) then it may not be a global square of an Abelian differential. But if all zeros are of even orders $k_{i}$ (except $\left(k_{1}, \ldots, k_{n}\right)=(\emptyset)$ or $\left.(4)\right)$ then the Theorem above says that there exist quadratic differentials with the singularity pattern $\left(k_{1}, \ldots, k_{n}\right)$ which are not squares of Abelian differentials. For example, on each complex curve of genus $g=2$, there exist quadratic differentials with two zeros of order two which are not squares of Abelian differentials (see also Figure 3), and there exist quadratic differentials with two zeros of order two which are the global squares of Abelian differentials. 
Theorem (H. Masur; W. Veech). Any stratum $Q\left(k_{1}, \ldots, k_{n}\right)$ is a complex orbifold of dimension

$$
\operatorname{dim}_{\mathbb{C}} \mathcal{Q}\left(k_{1}, \ldots, k_{n}\right)=2 g+n-2
$$

Remark 4. The principal stratum, that is all singularities of the form are only simples zeroes, can be identified with the cotangent fiber over the Teichmüller space. So the dimension in this case is

$$
2 \cdot \operatorname{dim}_{\mathbb{C}} T_{g}=6 g-6 .
$$

This corresponds to the above formula with $n=4 g-4$. The general formula is obtained by subtracting a dimension any time a zero is collapsed to higher order.

Proposition (Kontsevich). Any stratum $\mathcal{Q}\left(k_{1}, \ldots, k_{n}\right)$ with $\sum k_{i}=-4$ is connected.

Proof. Since there is only one complex structure on $\mathbb{C P}^{1}$ we can work in the standard atlas on $\mathbb{C P}^{1}=\mathbb{C} \cup\left(\mathbb{C}^{*} \cup \infty\right)$. In this atlas, we can easily find quadratic differentials $f(z)(d z)^{2}$ with any prescribed singularities at any prescribed points (with the evident condition prescribed by degrees of singularities, $\sum k_{i}=-4$ ) just by choosing an appropriate rational function $f(z)$. The space of configurations of points on a sphere is connected; this implies the statement of the Proposition.

Construction 1 (Canonical double covering). Let $M_{g}^{2}$ be a Riemann surface and let $\psi$ be a quadratic differential on it which is not a square of an Abelian differential. There exists a canonical (ramified) double covering $\pi: \tilde{M}_{\tilde{g}}^{2} \rightarrow M_{g}^{2}$ such that $\pi^{*} \psi=$ $\tilde{\omega}^{2}$, where $\tilde{\omega}$ is an Abelian differential on $\tilde{M}_{\tilde{g}}^{2}$.

The set of critical values of $\pi$ on $M_{g}^{2}$ coincide exactly with the set of singularities of odd degrees of $\psi$. The covering $\pi: \tilde{M}_{\tilde{g}}^{2} \rightarrow M_{g}^{2}$ is the minimal (ramified) covering such that the quadratic differential $\pi^{*} \psi$ becomes the square of an Abelian differential on $\tilde{M}_{\tilde{g}}^{2}$.

Proof. Consider an atlas $\left(U_{i}, z_{i}\right)_{i}$ on $\dot{M}_{g}^{2}=M_{g}^{2} \backslash\{$ singularities of $\psi\}$ where we punctured all the zeros and the poles of $\psi$. We assume that all the charts $U_{i}$ are connected and simply-connected. The quadratic differential $\psi$ can be represented in this atlas by a collection of holomorphic functions $f_{i}\left(z_{i}\right)$, where $z_{i} \in U_{i}$, satisfying the relations:

$$
f_{i}\left(z_{i}\left(z_{j}\right)\right) \cdot\left(\frac{d z_{i}}{d z_{j}}\right)^{2}=f_{j}\left(z_{j}\right) \text { on } U_{i} \cap U_{j} .
$$

Since we have punctured all singularities of $\psi$ any function $f_{i}\left(z_{i}\right)$ is nonzero at $U_{i}$. Consider two copies $U_{i}^{ \pm}$of every chart $U_{i}$ : one copy for every of two branches $g_{i}^{ \pm}\left(z_{i}\right)$ of $g^{ \pm}\left(z_{i}\right):=\sqrt{f_{i}\left(z_{i}\right)}$ (of course, the assignment of "+" or "-" is not canonical). Now for every $i$ identify the part of $U_{i}^{+}$corresponding to $U_{i} \cap U_{j}$ with 
the part of one of $U_{j}^{ \pm}$corresponding to $U_{j} \cap U_{i}$ in such way that on the overlap branches would match

$$
g_{i}^{+}\left(z_{i}\left(z_{j}\right)\right) \cdot \frac{d z_{i}}{d z_{j}}=g_{j}^{ \pm}\left(z_{j}\right) \text { on } U_{i}^{+} \cap U_{j}^{ \pm} .
$$

Apply the analogous identification to every $U_{i}^{-}$. We get a Riemann surface with punctures provided with a holomorphic 1 -form $\tilde{\omega}$ on it, where $\tilde{\omega}$ is presented by the collection of holomorphic functions $g_{i}^{ \pm}$in the local charts. It is an easy exercise to check that filling punctures we get a closed Riemann surface $\tilde{M}_{\tilde{g}}^{2}$, and that $\tilde{\omega}$ extends to an Abelian differential on it. We get a canonical (possibly ramified) double covering $\pi: \tilde{M}_{\tilde{g}}^{2} \rightarrow M_{g}^{2}$ such that $\pi^{*} \psi=\tilde{\omega}^{2}$.

By construction the only points of the base $M_{g}^{2}$ where the covering might be ramified are the singularities of $\psi$. In a small neighborhood of a zero of even degree $2 k$ of $\psi$ we can chose coordinates in which $\psi$ is presented as $z^{2 k}(d z)^{2}$. In this chart we get two distinct branches $\pm z^{k} d z$ of the square root. Thus the zeros of even degrees of $\psi$ and the marked points are the regular points of the covering $\pi$. However, it easy to see that the covering $\pi$ has a ramification point over any zero of odd degree and over any simple pole of $\psi$.

Cohomological coordinates. We use the construction above to describe the local cohomological coordinates on $\mathcal{Q}\left(k_{1}, \ldots, k_{n}\right)$ proposed by Kontsevich.

Let $[M, \psi] \in \mathcal{Q}\left(k_{1}, \ldots, k_{n}\right)$. Consider the canonical double covering $\pi: \tilde{M} \rightarrow$ $M$ described in above Construction 1 such that the pull-back $\pi^{*} \psi=\omega^{2}$ becomes the global square of an Abelian differential $\omega$ on $\tilde{M}$. Let $\tau$ be the natural involution of $\tilde{M}$ interchanging the points in the fibers of $\pi$. Let $\tilde{P}_{1}, \ldots, \tilde{P}_{r} \in \tilde{M}$ be the true zeros of $\omega$. Since by construction $\tau^{*} \omega=-\omega$, the set $\left\{\tilde{P}_{1}, \ldots, \tilde{P}_{r}\right\}$ is sent to itself by the involution $\tau$. Consider the induced involution

$$
\tau^{*}: H^{1}\left(\tilde{M},\left\{\tilde{P}_{1}, \ldots, \tilde{P}_{r}\right\} ; \mathbb{C}\right) \rightarrow H^{1}\left(\tilde{M},\left\{\tilde{P}_{1}, \ldots, \tilde{P}_{r}\right\} ; \mathbb{C}\right)
$$

of the relative cohomology group. The vector space $H^{1}\left(\tilde{M},\left\{\tilde{P}_{1}, \ldots, \tilde{P}_{r}\right\} ; \mathbb{C}\right)$ splits into direct sum

$$
H^{1}\left(\tilde{M},\left\{\tilde{P}_{1}, \ldots, \tilde{P}_{r}\right\} ; \mathbb{C}\right)=V_{1} \oplus V_{-1}
$$

of invariant and anti-invariant subspaces of the involution $\tau^{*}$. We have already seen that $[\omega] \in V_{-1}$. A small neighborhood of $[\omega]$ in the anti-invariant subspace $V_{-1}$ gives a local coordinate chart of a regular point $[M, \psi]$ (not a fixed point for a elliptic element of $\operatorname{Mod}(g))$ in the stratum $\mathcal{Q}\left(k_{1}, \ldots, k_{n}\right)$.

In what follows we consider ramified coverings $\pi: \tilde{M}_{\tilde{g}}^{2} \rightarrow M_{g}^{2}$ of arbitrary degree $d$. We denote the ramification index of $\pi$ at a point $\tilde{P} \in \tilde{M}_{\tilde{g}}^{2}$ by $e_{\pi}(\tilde{P})$. By convention we have $e_{\pi}(\tilde{P})=1$ at any regular point $\tilde{P}$ of the covering $\pi$. The Riemann-Hurwitz formula gives the value of the genus $\tilde{g}$ of the covering Riemann surface $\tilde{M}_{\tilde{g}}^{2}$. 
Riemann-Hurwitz Formula. Let $\pi: \tilde{M}_{\tilde{g}}^{2} \rightarrow M_{g}^{2}$ be an analytic map of degree $d$ between compact Riemann surfaces. The genus $\tilde{g}$ of $\tilde{M}_{\tilde{g}}^{2}$ and the genus $g$ of $M_{g}^{2}$ are related by the formula

$$
2 \tilde{g}-2=d \cdot(2 g-2)+\sum_{\tilde{P} \in \tilde{M}_{\tilde{g}}^{2}}\left(e_{\pi}(\tilde{P})-1\right)
$$

where $e_{\pi}(\tilde{P})$ is the index of the ramification of $\pi$ at $\tilde{P}$.

Having a ramified $d$-fold covering $\pi: \tilde{M}_{\tilde{g}}^{2} \rightarrow M_{g}^{2}$ and a meromorphic quadratic differential $\psi$ on $M_{g}^{2}$ with a singularity pattern $\left(k_{1}, \ldots, k_{n}\right)$ we will need to compute the singularity pattern $\left(\tilde{k}_{1}, \ldots, \tilde{k}_{m}\right)$ of the induced quadratic differential $\pi^{*} \psi$ on $\tilde{M}_{\tilde{g}}^{2}$.

Lemma 1. Let $\pi: \tilde{M}_{\tilde{g}}^{2} \rightarrow M_{g}^{2}$ be a (ramified) covering, and let $\psi$ be a meromorphic quadratic differential on $M_{g}^{2}$. A point $\tilde{P} \in \tilde{M}_{\tilde{g}}^{2}$ is a singular point of the induced quadratic differential $\pi^{*} \psi$ either if its image $P=\pi(\tilde{P})$ is a singular point of $\psi$ or if $\tilde{P}$ is a ramification point of $\pi$. The degree $\tilde{k}$ of $\pi^{*} \psi$ at the point $\tilde{P}$ and the degree $k$ of $\psi$ at the point $P=\pi(\tilde{P})$ are related as

$$
\tilde{k}=e_{\pi}(\tilde{P}) \cdot(k+2)-2 .
$$

Proof. Let $P \in M_{g}^{2}$ be a point of the base of the covering. Let the quadratic differential have a singularity of order $k$ at $P$. Recall that $k=-1$ if $P$ is a simple pole of $\psi$, and $k=0$ if $P$ is, actually, a regular point. Let $\tilde{P}$ be in the preimage $\pi^{-1}(P)$ of $P$. If $\tilde{P}$ is a regular point of the covering $\pi$ then $\pi^{*} \psi$ has at $\tilde{P}$ a singularity of the same degree $k$ as the singularity $P$ of $\psi$.

Suppose that $\tilde{P}$ is a ramification point of $\pi$ of index $e_{\pi}(\tilde{P})=b$. We can choose a local coordinate $z$ in the neighborhood of the point $P \in M_{g}^{2}$ in such way that $\psi=z^{k}(d z)^{2}$ in this coordinate. We can now chose the local coordinate $w$ in a neighborhood of $\tilde{P} \in \tilde{M}_{\tilde{g}}^{2}$ in such way that the projection $\pi$ has the form $z=w^{b}$ in this coordinate. Then we get the following representation of the induced quadratic differential $\pi^{*} \psi$ in the neighborhood of the point $\tilde{P}$

$$
\pi^{*} \psi=(z(w))^{k}(d(z(w)))^{2} \sim w^{b \cdot k}\left(w^{b-1} d w\right)^{2}=w^{b \cdot k+2 b-2}(d w)^{2} .
$$

In particular, if the order of the covering is 2 then a singularity of $\psi$ gives either one singularity of order $2 k+2$ or two singularities of order $k$ of $\pi^{*} \psi$.

We use the following obvious Corollary of Lemma 1.

Corollary 2. Let $\pi: \tilde{M}_{\tilde{g}}^{2} \rightarrow M_{g}^{2}$ be a ramified covering, and let $\psi$ be a meromorphic quadratic differential on $M_{g}^{2}$. A preimage $\tilde{P}=\pi^{-1}(P)$ of a singular point $P$ of $\psi$ is a regular point of $\pi^{*} \psi$ only in the following two cases 
- the ramification index $e_{\pi}(\tilde{P})$ of the point $\tilde{P}$ is equal to 2 , and $\psi$ has a simple pole at $P$;

- the ramification index $e_{\pi}(\tilde{P})$ of the point $\tilde{P}$ is equal to 1 and $P$ is a fake zero of $\psi$.

To complete this section we present a construction of a natural mapping of the strata induced by a ramified covering of the fixed combinatorial type. This mapping was introduced in [KoZo] to construct the hyperelliptic connected components of the moduli spaces of Abelian differentials. In the next section we shall use this mapping to construct the hyperelliptic connected components of the moduli spaces of quadratic differentials.

Let $M_{g}^{2}$ be a Riemann surface and let $\psi_{0}$ be a quadratic differential on it which is not a square of an Abelian differential. Let $\left(k_{1}, \ldots, k_{n}\right)$ be its singularity pattern. We do not exclude the case when some of $k_{i}$ are equal to zero: by convention this means that we have some marked points.

Let $\pi: \tilde{M}_{\tilde{g}}^{2} \rightarrow M_{g}^{2}$ be a (ramified) covering such that the image of any ramification point of $\pi$ is a marked point, or a zero, or a pole of the quadratic differential $\psi_{0}$. Fix the combinatorial type of the covering $\pi$ : the degree of the covering, the number of critical fibers and the ramification index of the points in every critical fiber. Consider the induced quadratic differential $\pi^{*} \psi_{0}$ on $\tilde{M}_{\tilde{g}}^{2}$; let $\left(\tilde{k}_{1}, \ldots, \tilde{k}_{m}\right)$ be its singularity pattern.

Construction 2 (Ramified covering construction). Deforming slightly the initial point $\left[M_{g}^{2}, \psi_{0}\right] \in \mathcal{Q}\left(k_{1}, \ldots, k_{n}\right)$ we can consider a ramified covering over the deformed Riemann surface of the same combinatorial type as the covering $\pi$. This new covering has exactly the same relation between the position and types of the ramification points and the degrees and position of the singularities of the deformed quadratic differential. This means that the induced quadratic differential $\pi^{*} \psi$ has the same singularity pattern $\left(\tilde{k}_{1}, \ldots, \tilde{k}_{m}\right)$ as $\pi^{*} \psi_{0}$. Thus we get a local mapping

$$
\begin{aligned}
\mathcal{Q}\left(k_{1}, \ldots, k_{n}\right) & \rightarrow \mathcal{Q}\left(\tilde{k}_{1}, \ldots, \tilde{k}_{m}\right) \\
{\left[M_{g}^{2}, \psi\right] } & \mapsto\left(\tilde{M}_{g}^{2}, \pi^{*} \psi\right) .
\end{aligned}
$$

Note that in general the corresponding global mapping is multi-valued. For example, if there is a pair of singularities of $\psi$ of the same order $k$ such that the first one is the image of a ramification point of the covering and the other one is not, then the corresponding map is multi-valued.

Note also that for ramified coverings of some special types the image of the mapping belongs to a stratum of squares of Abelian differentials. This is the case, for example, for the canonical double covering described in Construction 1.

"Forgetful" map. Consider a stratum of meromorphic quadratic differentials 
with $m$ marked points

$$
\mathcal{Q}\left(k_{1}, \ldots, k_{n}, k_{n+1}, \ldots, k_{n+m}\right)
$$

where $k_{i} \neq 0$ for $i=1, \ldots, n$ and $k_{i+n}=0$ for $i=1, \ldots, m$. We have the following forgetful map

$$
\mathcal{Q}\left(k_{1}, \ldots, k_{n}, k_{n+1}, \ldots, k_{n+m}\right) \rightarrow \mathcal{Q}\left(k_{1}, \ldots, k_{n}\right)
$$

Remark 5. We are interested by the topology of the strata, that is the classification of connected components of strata $\mathcal{Q}\left(k_{1}, \ldots, k_{n}\right)$ with all $k_{i} \neq 0$. One can prove that the topology of strata with marked points and the topology of strata without marked point coincide. Nevertheless we use strata with marked point, that is some $k_{i}$ is equal to zero, for the above construction.

We use Construction 2 to obtain connected components of the strata with no marked points. Thus we use, actually, the composition of the mapping defined above with the forgetful map.

Convention. By convention we consider only those coverings $\pi: \tilde{M} \rightarrow M$, that have at least one ramification point in the fiber over any marked point of $\psi$ on the underlying surface.

- We shall call the images of ramification points the critical values of $\pi$. Our convention means that the marked points of $\psi$ form a subset of the critical values of $\pi$.

We complete this section with the following statement.

Lemma 2. With the above convention the mapping

$$
\mathcal{Q}\left(k_{1}, \ldots, k_{n}\right) \rightarrow \mathcal{Q}\left(\tilde{k}_{1}, \ldots, \tilde{k}_{m}\right)
$$

is locally an embedding.

Proof of Lemma 2. We need to prove that the mapping constructed above is locally injective near a regular point $[M, \psi]$ in $\mathcal{Q}\left(k_{1}, \ldots, k_{n}\right)$. We use the cohomological coordinates (see above) in the neighborhood of $[M, \psi] \in \mathcal{Q}\left(k_{1}, \ldots, k_{n}\right)$ and in the neighborhood of its image $[\tilde{M}, \tilde{\psi}] \in \mathcal{Q}\left(\tilde{k}_{1}, \ldots, \tilde{k}_{m}\right)$, where $\tilde{\psi}=\pi^{*} \psi$. These coordinates linearize the mapping, and the proof becomes an exercise in algebraic topology.

Take the two canonical double coverings (see Construction 1)

$$
p: N \rightarrow M \quad \text { and } \quad \tilde{p}: \tilde{N} \rightarrow \tilde{M}
$$

such that the pull-back $p^{*} \psi=\omega^{2}$ of the quadratic differential $\psi$ from $M$ to $N$ becomes the global square of an Abelian differential $\omega$ on $N$, and the pull-back $\tilde{p}^{*} \tilde{\psi}=\tilde{\omega}^{2}$ of the quadratic differential $\tilde{\psi}$ from $\tilde{M}$ to $\tilde{N}$ becomes the global square of an Abelian differential $\tilde{\omega}$ on $\tilde{N}$. If the quadratic differential $\tilde{\psi}$ is already the 
global square of an Abelian differential on $\tilde{M}$, the double covering $\tilde{p}: \tilde{N} \rightarrow \tilde{M}$ is not connected: it is composed of two copies of $\tilde{M}$, and the corresponding Abelian differential on $\tilde{N}$ corresponds to two branches $\pm \tilde{\omega}$ of the globally defined square root of $\tilde{\psi}$.

It follows from the definition of the canonical double covering, that the diagram

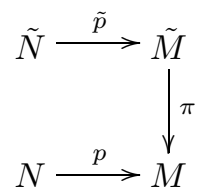

can be completed to a commutative diagram

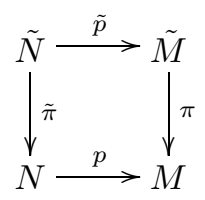

Moreover, the induced (ramified) covering $\tilde{\pi}: \tilde{N} \rightarrow N$ can be chosen in such way that $\tilde{\omega}=\tilde{\pi}^{*} \omega$. In particular, it intertwines the natural involutions $\tilde{\tau}: \tilde{N} \rightarrow \tilde{N}$ and $\tau: N \rightarrow N$, that is $\tilde{\pi} \circ \tilde{\tau}=\tau \circ \tilde{\pi}$.

Denote by $Q_{1}, \ldots, Q_{r}$ the zeroes of $\omega$ on $N$. The set $\left\{Q_{1}, \ldots, Q_{r}\right\}$ is obtained as an inverse image $p^{-1}$ of the set of true zeros, the marked points and the poles of $\psi$.

Note that we do not mark any points at $\tilde{M}$ (we are interested by classification of strata without marked point, that is without fake singularities). Then the Convention draw aside the trivial cases when the map is degenerated.

Denote by $\tilde{Q}_{1}, \ldots, \tilde{Q}_{l}$ the zeros of $\tilde{\omega}$ on $\tilde{N}$. Since $\tilde{\omega}=\tilde{\pi}^{*} \omega$, we see that any zero $\tilde{Q}_{i}$ of $\tilde{\omega}$ located at a regular point of the covering $\tilde{\pi}$ is projected by $\tilde{\pi}$ to a zero of $\omega$. It follows from Convention that the image of a zero $\tilde{Q}_{j}$ of $\tilde{\omega}$ located at a ramification point of the covering $\tilde{\pi}$ is also projected by $\tilde{\pi}$ to a zero or to a marked point of $\omega$. Thus

$$
\tilde{\pi}\left(\left\{\tilde{Q}_{1}, \ldots, \tilde{Q}_{l}\right\}\right) \subset\left\{Q_{1}, \ldots, Q_{r}\right\} .
$$

Hence the covering map $\tilde{\pi}$ induces a mapping

$$
\tilde{\pi}^{*}: H^{1}\left(N,\left\{Q_{1}, \ldots, Q_{r}\right\}, \mathbb{C}\right) \rightarrow H^{1}\left(\tilde{N},\left\{\tilde{Q}_{1}, \ldots, \tilde{Q}_{l}\right\}, \mathbb{C}\right) .
$$

Since this mapping intertwines the natural involutions $\tilde{\pi}^{*} \circ \tilde{\tau}^{*}=\tau^{*} \circ \tilde{\pi}^{*}$, the subset $V_{-1} \subset H^{1}\left(N,\left\{Q_{1}, \ldots, Q_{r}\right\}, \mathbb{C}\right)$ anti-invariant under the involution $\tau^{*}$ is mapped into the subset $\tilde{V}_{-1} \subset H^{1}\left(\tilde{N},\left\{\tilde{Q}_{1}, \ldots, \tilde{Q}_{l}\right\}, \mathbb{C}\right)$ anti-invariant under the involution $\tilde{\tau}^{*}$. The induced map $\tilde{\pi}^{*}: V_{-1} \rightarrow \tilde{V}_{-1}$ restricted to an appropriate neighborhoods of $[M, \psi] \in V_{-1}$ and $[\tilde{M}, \tilde{\psi}] \in \tilde{V}_{-1}$ coincides with the mapping $\mathcal{Q}\left(k_{1}, \ldots, k_{n}\right) \rightarrow \tilde{\mathcal{Q}}\left(\tilde{k}_{1}, \ldots, \tilde{k}_{m}\right)$ written down in the cohomological coordinates.

It is obvious that the map of the absolute cohomology groups

$$
\tilde{\pi}^{*}: H^{1}(N ; \mathbb{C}) \rightarrow H^{1}(\tilde{N} ; \mathbb{C})
$$


induced by the covering $\pi: \tilde{N} \rightarrow N$ is a monomorphism. It is not difficult to see that, with our convention on the covering $\pi$, the restriction of the mapping $\tilde{\pi}^{*}: V_{-1} \rightarrow \tilde{V}_{-1}$ to the anti-invariant subspace of $\tau^{*}$ is also a monomorphism, which completes the proof of Lemma 2. Note that in general, the mapping $\tilde{\pi}^{*}$ defined on the relative cohomology group is not a monomorphism.

\section{Hyperelliptic components}

\subsection{The Teichmüller geodesic flow}

The group $G L(2, \mathbb{R})_{+}$acts naturally on the moduli space of quadratic differentials; this action preserves the natural stratification of the moduli space.

The action of the diagonal subgroup of $S L(2, \mathbb{R})$ on the moduli space of quadratic differentials can be naturally identified with the Teichmüller geodesic flow on the moduli space of curves for the Teichmüller metric.

Many important results in the theory of interval exchange maps, of measured foliations and of dynamics on translation surfaces are based on the following fundamental Theorem, proved independently by H. Masur [Ma] and by W. Veech [Ve1]:

Theorem (H. Masur; W. Veech). The Teichmüller geodesic flow acts ergodically on every connected component of every (normalized) stratum of the moduli space of quadratic differentials; the corresponding invariant measure is a finite Lebesque equivalent measure.

Proposition 1. The action of $G L(2, \mathbb{R})_{+}$on the strata commutes with the mapping of Construction 2.

Proof. In the proof of Lemma 2, we use some coordinates to linearize the mapping. It is easy to see that in these charts, the linear action commute with the mapping.

\subsection{Hyperelliptic components}

Let us apply Construction 2 in the following particular case.

Consider a meromorphic quadratic differential $\psi$ on $\mathbb{C P}^{1}$ having the singularity pattern $\left(2(g-k)-3,2 k+1,-1^{2 g+2}\right)$, where $k \geq-1, g \geq 1$ and $g-k \geq 2$. Consider a ramified double covering $\pi$ over $\mathbb{C P}^{1}$ having ramification points over $2 g+2$ poles of $\psi$, and no other ramification points. We obtain a hyperelliptic Riemann surface $\tilde{M}$ of genus $g$ with a quadratic differential $\pi^{*} \psi$ on it. By Lemma 1 the induced quadratic differential $\pi^{*} \psi$ has the singularity pattern $(2(g-k)-3,2(g-k)-$ $3,2 k+1,2 k+1)$. Construction 2 gives us a local mapping

$$
\mathcal{Q}\left(2(g-k)-3,2 k+1,-1^{2 g+2}\right) \rightarrow \mathcal{Q}(2(g-k)-3,2(g-k)-3,2 k+1,2 k+1),
$$


where $k \geq-1, g \geq 1$ and $g-k \geq 2$. Computing the dimensions of the strata we get

$$
\begin{aligned}
& \operatorname{dim}_{\mathbb{C}} \mathcal{Q}\left(2(g-k)-3,2 k+1,-1^{2 g+2}\right)=2 \cdot 0+(2 g+4)-2=2 g+2 \\
& \operatorname{dim}_{\mathbb{C}} \mathcal{Q}(2(g-k)-3,2(g-k)-3,2 k+1,2 k+1)=2 g+4-2=2 g+2 .
\end{aligned}
$$

Thus the dimensions of the strata coincide. By Lemma 2 the mapping is nondegenerate.

When $2 k+1 \neq-1$ the mapping

$$
\mathcal{Q}\left(2(g-k)-3,2 k+1,-1^{2 g+2}\right) \rightarrow \mathcal{Q}(2(g-k)-3,2(g-k)-3,2 k+1,2 k+1)
$$

is globally defined. Since the stratum $\mathcal{Q}\left(2(g-k)-3,2 k+1,-1^{2 g+2}\right)$ is connected, as any other stratum on $\mathbb{C P}^{1}$ by Proposition in section 2 , see above, the image of the mapping in the stratum $\mathcal{Q}(2(g-k)-3,2(g-k)-3,2 k+1,2 k+1)$ is also connected. When $2 k+1=-1$ the mapping has $2 g+3$ branches corresponding to the choice of the simple pole of $\psi$ where we do not have a ramification of the covering. However, since we can deform the positions of the zero and the poles of $\psi$ on $\mathbb{C P}^{1}$ arbitrarily (avoiding collapses, of course), the intersection of the image is non empty, so the union of the images of the mapping $\mathcal{Q}\left(2 g-1,-1^{2 g+3}\right) \rightarrow \mathcal{Q}(2 g-1,2 g-1,-1,-1)$ is connected.

Since the dimension of the strata coincide, and the mapping is non-degenerate, we obtain an open set on the stratum $\mathcal{Q}(2(g-k)-3,2(g-k)-3,2 k+1,2 k+1)$. By Proposition 1, the action of the geodesic flow is relevant, thus by ergodicity of this flow, the image of the mapping

$$
\mathcal{Q}\left(2(g-k)-3,2 k+1,-1^{2 g+2}\right) \rightarrow \mathcal{Q}(2(g-k)-3,2(g-k)-3,2 k+1,2 k+1)
$$

gives us a full measure set in the corresponding connected component of the stratum

$$
\mathcal{Q}(2(g-k)-3,2(g-k)-3,2 k+1,2 k+1) .
$$

Thus we obtain a connected component of these stratum.

Similarly to the previous case we can easily check coincidence of the dimensions of the strata

$$
\mathcal{Q}\left(2(g-k)-3,2 k,-1^{2 g+1}\right) \rightarrow \mathcal{Q}(2(g-k)-3,2(g-k)-3,4 k+2),
$$

with $k \geq 0, g \geq 1$ and $g-k \geq 1$ and

$$
\mathcal{Q}\left(2 g-2 k-4,2 k,-1^{2 g}\right) \rightarrow \mathcal{Q}(4(g-k)-6,4 k+2)
$$

with $k \geq 0, g \geq 2$ and $g-k \geq 2$.

The images of these mappings give us connected components in the strata

$$
\mathcal{Q}(2(g-k)-3,2(g-k)-3,4 k+2) .
$$

and

$$
\mathcal{Q}(4(g-k)-6,4 k+2)
$$


Definition 1. The connected components constructed above are called the hyperelliptic components and are denoted by:

(1) $\mathcal{Q}\left(2(g-k)-3,2 k+1,-1^{2 g+2}\right) \rightarrow \mathcal{Q}^{h y p}(2(g-k)-3,2(g-k)-3,2 k+1,2 k+1)$, where $k \geq-1, g \geq 1, g-k \geq 2$. The corresponding double covering has ramification points over $2 g+2$ poles of meromorphic quadratic differential on $\mathbb{C P}^{1}$

(2) $\mathcal{Q}\left(2(g-k)-3,2 k,-1^{2 g+1}\right) \rightarrow \mathcal{Q}^{\text {hyp }}(2(g-k)-3,2(g-k)-3,4 k+2)$, where $k \geq 0, g \geq 1$ and $g-k \geq 1$. The corresponding double covering has ramification points over $2 g+1$ poles and over the zero of degree $2 k$ of meromorphic quadratic differential on $\mathbb{C P}^{1}$.

(3) $\mathcal{Q}\left(2 g-2 k-4,2 k,-1^{2 g}\right) \rightarrow \mathcal{Q}^{h y p}(4(g-k)-6,4 k+2)$, where $k \geq 0, g \geq 2$ and $g-k \geq 2$. The corresponding double covering has ramification points over all singularities of the quadratic differential on $\mathbb{C P}^{1}$.

Remark 6. The connected component $\mathcal{Q}^{h y p}(2(g-k)-3,2(g-k)-3,2 k+1,2 k+1)$ was first noticed by M. Kontsevich.

Remark 7. For the mapping

$$
\mathcal{Q}\left(2 g-2 k-4,2 k,-1^{2 g}\right) \rightarrow \mathcal{Q}(4(g-k)-6,4 k+2)
$$

one can see that the resulting quadratic differential is not the global square of an Abelian differential in the following way. Take a path $\gamma$ on $\mathbb{C P}^{1}$ around the zero of order $2 k$ and a pole of the corresponding quadratic differential. The monodromy of the covering along this path is trivial so it can be lifted on a path $\tilde{\gamma}$ in $M$. The holonomy of the flat structure along the path $\tilde{\gamma}$ is non trivial, we can compute how the tangent vector to the corresponding path $\tilde{\gamma}$ on $M$ turns in the flat structure defined by $\psi$ (see Figure 1). The counterclockwise direction is chosen as a direction of the positive turn.

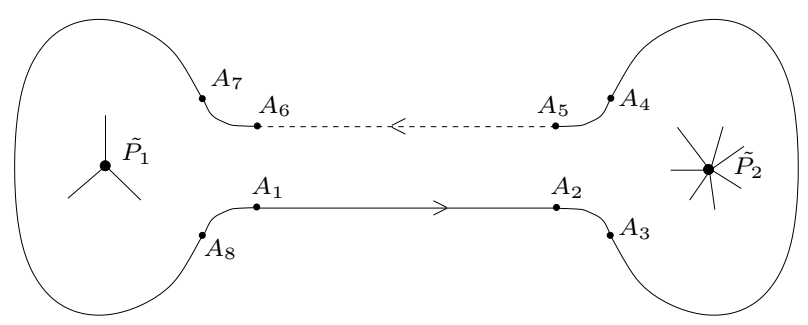

FIG. 1. A path $\tilde{\gamma}$ on the covering surface obtained by lifting of the path $\gamma$. Since $\tilde{P}_{1}, \tilde{P}_{2} \in \tilde{M}$ are ramification points, the segments $A_{1} A_{2}$ and $A_{6} A_{5}$ are located at the "different sheets" of the covering $\tilde{M} \rightarrow M$, and not nearby.

We get some angle $\phi$ following the part $A_{1} A_{2}$ of the path which goes from one 
singularity to another. Then we make a turn by $-\pi / 2$ going along $A_{2} A_{3}$. Turning around the singularity (the path $\left.A_{3} A_{4}\right)$ we get the angle $\left(k_{2}+2\right) \pi$, which is followed by another turn by $-\pi / 2$ now along $A_{4} A_{5}$. The path $A_{5} A_{6}$ gives the turn by $-\phi$, which is followed by another $-\pi / 2$ along $A_{6} A_{7}$. Turning around singularity along $A_{7} A_{8}$ we get the angle $\left(k_{1}+2\right) \pi$, and the loop is competed by the path $A_{8} A_{1}$ giving one more turn by $-\pi / 2$. All together this gives $\left(k_{1}+k_{2}+2\right) \cdot \pi$.

In our special case, the resulting angle for the corresponding quadratic differential is $(2 k+1) \cdot \pi$, thus the corresponding measured foliation is not oriented and consequently, the quadratic differential $\psi$ on $M$ is not the global square of an Abelian differential. The image of application above belong in strata $\mathcal{Q}(4(g-k)-6,4 k+2)$ and the application above is well defined.

Remark 8. In the each of the following cases

$$
\begin{aligned}
& \mathcal{Q}(-1,-1,-1,-1,0,0) \rightarrow \mathcal{Q}(2,2) \\
& \mathcal{Q}(-1,-1,-1,-1,0) \rightarrow \mathcal{Q}(-1,-1,2) \\
& \mathcal{Q}(-1,-1,-1,-1) \rightarrow \mathcal{Q}(-1,-1,-1,-1)
\end{aligned}
$$

the image stratum also has the same dimension as the original one. All the corresponding ramified coverings have even degree $2 d \geq 2$.

In the first case the covering has $d$ ramification points of degree 2 over each of four simple poles of the meromorphic quadratic differential on $\mathbb{C P}^{1}$; a single ramification point of degree 2 over each of two marked points, and no other ramification points.

In the second case the covering has $d-1$ ramification points of degree 2 over one of the poles; $d$ ramification points of degree 2 over each of the remaining three simple poles of the meromorphic quadratic differential on $\mathbb{C P}^{1}$; a single ramification point of degree 2 over the marked point, and no other ramification points.

In the third case the covering has $d-1$ ramification points of degree 2 over each of two poles; $d$ ramification points of degree 2 over each of remaining two simple poles of the meromorphic quadratic differential on $\mathbb{C P}^{1}$, and no other ramification points.

The strata $\mathcal{Q}(-1,-1,2)$ and $\mathcal{Q}(2,2)$ are in the list of hyperelliptic components in the definition above; the corresponding surfaces of genus 1 and 2 are respectively elliptic and hyperelliptic. We show in section 4, Lemma 7 that theses strata are connected, which implies that the corresponding mappings have no interest for our purposes. The stratum $\mathcal{Q}(-1,-1,-1,-1)$ corresponds to quadratic differentials on $\mathbb{C P}^{1}$ so it is connected by Proposition of Kontsevich. Thus the third mapping is of no interest for us neither.

The Theorem below asserts that there are no other connected components which can be obtained using a similar construction.

Theorem 1. Let $\mathcal{Q}\left(k_{1}, \ldots, k_{n}\right)$ be a stratum in the moduli space of meromorphic quadratic differentials and let $\pi: \tilde{M} \rightarrow M$ be a covering of finite degree $d>1$. 
Consider the mapping

$$
\mathcal{Q}\left(k_{1}, \ldots, k_{n}\right) \rightarrow \mathcal{Q}\left(\tilde{k}_{1}, \ldots, \tilde{k}_{m}\right)
$$

induced by the covering $\pi$ (see Construction 2). Suppose that the image stratum is not a stratum of squares of Abelian differentials, and suppose that the mapping is neither of one of the three types corresponding to hyperelliptic components nor of one of the three exceptional types (2). Then

$$
\operatorname{dim}_{\mathbb{C}} \mathcal{Q}\left(k_{1}, \ldots, k_{n}\right)<\operatorname{dim}_{\mathbb{C}} \mathcal{Q}\left(\tilde{k}_{1}, \ldots, \tilde{k}_{m}\right)
$$

Proof. We introduce the integer parameters $d, n, m, p, r$ responsible for the topological type of the pair: (covering $\pi$, quadratic differential $\psi$ ). Let $d$ denote the degree of the covering, $n$ the number of true zeros which are critical values, $m$ the number of marked points, $p$ the number of simple poles, $r$ the number of singularities of $\psi$ which are regular values for the covering $\pi$. See below for an explicit example and details.

In the first part of the proof we derive from the relation $\operatorname{dim} \mathcal{Q}=\operatorname{dim} \tilde{\mathcal{Q}}$ elementary inequality (9) for the positive integers $d, n, m, p, r$. The inequality has not so many solutions. In Lemma 3 we show that solutions with $d \geq 3$, where $d$ stands for the number of branches of the covering $\pi$, do not correspond to any nontrivial mappings $\mathcal{Q} \rightarrow \tilde{\mathcal{Q}}$. In Lemma 4 we show that the only two-fold coverings $\pi$ which give rise to the solution of the equation $\operatorname{dim} \mathcal{Q}=\operatorname{dim} \tilde{\mathcal{Q}}$ are exactly those which correspond to hyperelliptic components.

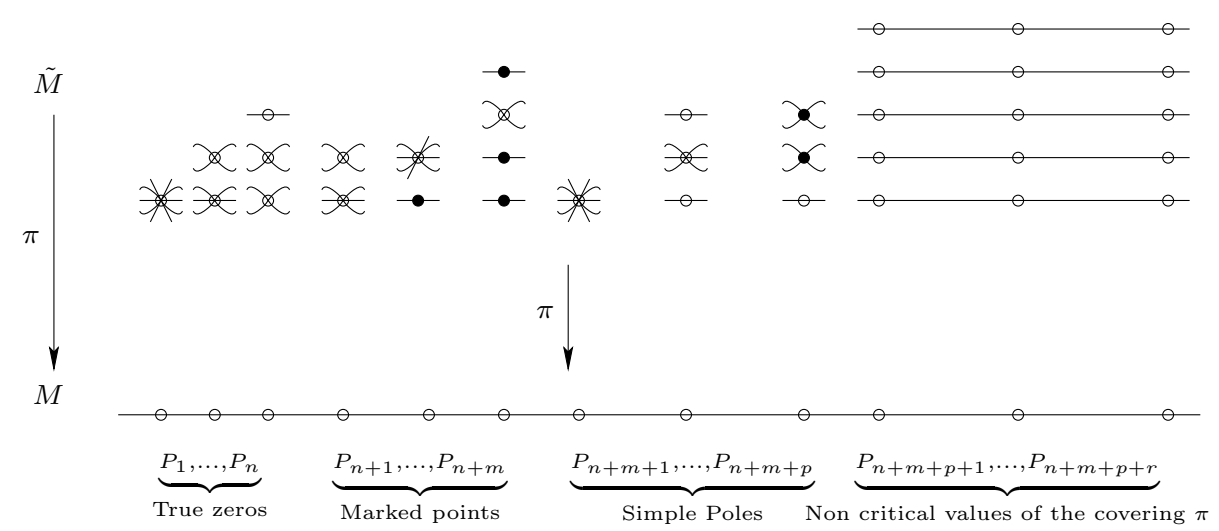

FIG. 2. This example presents a covering of degree 5 with 21 ramification points. There are 9 critical values of $\pi$. Here we have $n=m=p=r=3$. The points $\tilde{P} \in \pi^{-1}\left(P_{i}\right)$ which correspond to regular points of $\pi^{*} \psi$ are painted in black. By convention we do not mark them.

Let us first introduce some notations. By $(M, \psi)$ we denote the initial Riemann surface and a quadratic differential on it. We consider a ramified covering $\pi: \tilde{M} \rightarrow$ $M$ of degree $d$. We denote by $P_{1}, \ldots, P_{n} \in M$ be the critical values corresponding 
to the true zeros of $\psi$, i.e. to the zeros of degrees $k_{i}>0$. Let $P_{n+1}, \ldots, P_{n+m} \in M$ be the critical values corresponding to the marked points (or, in the other words, to the fake zeros of $\psi$ ). Let $P_{n+m+1}, \ldots, P_{n+m+p} \in M$ be the critical values corresponding to the simple poles of $\psi$. Let $P_{n+m+p+1}, \ldots, P_{n+m+p+r} \in M$ be the remaining true zeros and simple poles of $\psi$ which correspond to non-critical values of $\pi$. (See an example at Figure 2).

We consider only those ramified coverings $\pi$ for which the induced quadratic differential $\tilde{\psi}=\pi^{*} \psi$ on $\tilde{M}$ is not globally a square of a holomorphic 1-form. We do not exclude from consideration the situation when the covering $\pi$ is actually a regular covering.

Let us discuss now the location of singularities of the quadratic differential $\tilde{\psi}=\pi^{*} \psi$ on $\tilde{M}$. By convention we do not endow $\tilde{\psi}$ with any fake zeros: all singularities of $\tilde{\psi}$ are either true zeros, or simple poles.

The computation of Lemma 1 shows that $\tilde{\psi}$ may have a singularity at a point $\tilde{P} \in \tilde{M}$ either when $\psi$ has a singularity at $\pi(\tilde{P})$ or when $\tilde{P}$ is a ramification point of $\pi$. Thus all zeros and poles of $\tilde{\psi}$ are located at the preimage of the points $P_{1}, \ldots, P_{n+m+p+r}$. Let us specify them precisely. (See also Figure 2 where singularities are denoted by white bullets.)

At any preimage of any of the zeros $P_{1}, \ldots, P_{n}$ of $\psi$ we have a true zero of $\tilde{\psi}$, see Lemma 1. We have a zero of $\tilde{\psi}$ at a preimage $\tilde{P} \in \pi^{-1}\left(P_{i}\right)$ of a marked point $P_{i}, n+1 \leq i \leq n+m$, if and only if $\tilde{P}$ is a ramification point of the covering $\pi$, $e_{\pi}(\tilde{P}) \neq 1$. We have a pole or a true zero of $\tilde{\psi}$ at a preimage $\tilde{P} \in \pi^{-1}\left(P_{i}\right)$ of a simple pole $P_{i}, n+m+1 \leq i \leq n+m+p$, if and only if the ramification index $e_{\pi}(\tilde{P})$ of the covering $\pi$ at $\tilde{P}$ is different from two, $e_{\pi}(\tilde{P}) \neq 2$. At any preimage of any singularity $P_{n+m+p+1}, \ldots, P_{n+m+p+r}$ of $\psi$ we have a simple pole or a true zero of $\tilde{\psi}$. (See also an example presented at Figure 2.)

Now everything is ready for the computation of dimensions of the strata of meromorphic quadratic differentials $\mathcal{Q}$ and $\tilde{\mathcal{Q}}$ corresponding to $\psi$ and $\tilde{\psi}$.

Let $g$ be the genus of the Riemann surface $M$. We have

$$
\operatorname{dim} \mathcal{Q}=2 g+n+m+p+r-2 .
$$

Denoting by $\tilde{g}$ be the genus of the Riemann surface $\tilde{M}$ we get

$$
\begin{aligned}
\operatorname{dim} \tilde{\mathcal{Q}}=2 \tilde{g} & + \text { number of singularities of } \tilde{\psi}-2 \\
=2 \tilde{g} & +\sum_{i=1}^{n} \text { number of preimage } \tilde{P} \text { of } \pi^{-1}\left(P_{i}\right) \\
& +\sum_{i=n+1}^{n+m} \text { number of preimage } \tilde{P} \text { of } \pi^{-1}\left(P_{i}\right) \text { of index } e_{\pi}(\tilde{P}) \neq 1 \\
& +\sum_{i=n+m+1}^{n+m+p} \text { number of preimage } \tilde{P} \text { of } \pi^{-1}\left(P_{i}\right) \text { of index } e_{\pi}(\tilde{P}) \neq 2
\end{aligned}
$$




$$
\begin{gathered}
+\sum_{i=n+m+p+1}^{n+m+p+r} \text { number of preimage } \tilde{P} \text { of } \pi^{-1}\left(P_{i}\right)-2 \\
=(2 \tilde{g}-2)+d \cdot r+\sum_{i=1}^{n+m+p} \text { number of preimage } \tilde{P} \text { in } \pi^{-1}\left(P_{i}\right) \\
\quad-\sum_{i=n+1}^{n+m} \text { number of preimage } \tilde{P} \text { in } \pi^{-1}\left(P_{i}\right) \text { of ramification index one } \\
\quad-\sum_{i=n+m+1}^{n+m+p} \text { number of preimage } \tilde{P} \text { in } \pi^{-1}\left(P_{i}\right) \text { of ramification index two. }
\end{gathered}
$$

Computing the genus $\tilde{g}$ of the Riemann surface $\tilde{M}$ by Riemann-Hurwitz formula

$$
\begin{aligned}
2 \tilde{g}-2 & =d \cdot(2 g-2)+\sum_{\text {ramification points }}\left(e_{\pi}(P)-1\right) \\
& =d \cdot(2 g-2)+\sum_{\text {critical values } P_{i} \text { of } \pi}(d-\text { number of preimage }) \\
& =d \cdot(2 g-2)+d \cdot(n+m+p)-\sum_{i=1}^{n+m+p} \text { number of preimage } \tilde{P} \text { in } \pi^{-1}\left(P_{i}\right)
\end{aligned}
$$

and substituting $2 \tilde{g}-2$ in the formula (4) by the latter expression we get the following answer:

$$
\begin{aligned}
\operatorname{dim} \tilde{\mathcal{Q}} & =d \cdot(2 g+n+m+p+r-2) \\
& -\sum_{i=n+1}^{n+m} \text { number of preimage } \tilde{P} \text { in } \pi^{-1}\left(P_{i}\right) \text { of ramification index one } \\
& -\sum_{i=n+m+1}^{n+m+p} \text { number of preimage } \tilde{P} \in \pi^{-1}\left(P_{i}\right) \text { of ramification index two. }
\end{aligned}
$$

Comparing the $\operatorname{dimensions} \operatorname{dim} \mathcal{Q}$ and $\operatorname{dim} \tilde{Q}$ given by equations (3) and (5) we see that the equation

$$
\operatorname{dim} \mathcal{Q}=\operatorname{dim} \tilde{Q}
$$

is equivalent to the following one

$$
\begin{aligned}
& (d-1) \cdot(2 g+n+m+p+r-2) \\
& \quad=\sum_{i=n+1}^{n+m} \text { number of preimage } \tilde{P} \text { in } \pi^{-1}\left(P_{i}\right) \text { of ramification index one }
\end{aligned}
$$




$$
+\sum_{i=n+m+1}^{n+m+p} \text { number of preimage } \tilde{P} \in \pi^{-1}\left(P_{i}\right) \text { of ramification index two. }
$$

By convention $P_{n+1}, \ldots, P_{n+m} \in M$ are critical values of the $d$-fold covering $\pi$. Hence, among preimage of any $\tilde{P}$ in $\pi^{-1}\left(P_{i}\right), n+1 \leq i \leq n+m$ there is at least one preimage with ramification index different from one. This implies that the number of preimage $\tilde{P}$ in $\pi^{-1}\left(P_{i}\right)$ of ramification index one is at most $d-2$.

The covering $\pi: \tilde{M} \rightarrow M$ is of order $d$. Hence, for any point $P_{i} \in M$ the number of preimage $\tilde{P} \in \pi^{-1}\left(P_{i}\right)$ of ramification index two has the following obvious upper bound

number of preimage $\tilde{P}$ in $\pi^{-1}\left(P_{i}\right)$ of ramification index two

$$
\leq \begin{cases}d / 2 & \text { when } d \text { is even } \\ (d-1) / 2 & \text { when } d \text { is odd }\end{cases}
$$

Using these two obvious bounds we derive the following inequality from equation $(6)$

$$
(d-1) \cdot(2 g+n+m+p+r-2) \leq \begin{cases}m \cdot(d-2)+p \cdot d / 2 & \text { when } d \text { is even } \\ m \cdot(d-2)+p \cdot(d-1) / 2 & \text { when } d \text { is odd }\end{cases}
$$

and its weakened version

$$
(d-1) \cdot(2 g-2+n+r) \leq p \cdot(1-d / 2)-m .
$$

In what follows we consider the nonnegative integer solutions of inequalities (8) and (9). There are not so many of them since for most types of the covering $\pi$ the dimension $\operatorname{dim} \tilde{Q}$ is greater than $\operatorname{dim} \mathcal{Q}$ and the parameters $d, n, m, p, r$ do not obey neither equation (6) nor even inequalities (8) and (9). In Lemma 3 we show that the solutions of inequalities with $d \geq 3$ do not correspond to any actual ramified coverings $\pi$. In Lemma 4 we study solutions of inequalities corresponding to $d=2$, and show that the two-fold coverings $\pi$ giving solutions of equation (6) are exactly those which corresponding to hyperelliptic components.

Lemma 3. If $d \geq 3$ then $\operatorname{dim} \mathcal{Q}<\operatorname{dim} \tilde{\mathcal{Q}}$.

Proof of Lemma 3. Suppose that dimensions of $\mathcal{Q}$ and $\tilde{\mathcal{Q}}$ coincide. We can apply the inequalities above.

First of all, we are going to show that genus $g$ of the underlying surface $M$ must be equal to 0 . Suppose that $g \geq 1$, so inequality (9) gives $(d-1)(n+r) \leq-p / 2-m$. If $p>0$ or $m>0$ then $n+r$ must be negative, which is impossible. If $p=0$ and $m=0$ then $n=r=p=m=0$ and we obtain the stratum $\mathcal{Q}(\emptyset)$ which is empty. Thus the assumption $g \geq 1$ leads to a contradiction and we must have $g=0$ which means that $M \simeq \mathbb{C P}$. 
Now suppose that $m>0$. Since the right-hand side of inequality (9) is strictly negative, and $d-1>0$, the expression $(2 g-2+n+r)$ should be strictly negative. Since $g=0$ this implies that

$$
n+r \leq 1 \text {. }
$$

Now suppose that $m=0$. Using inequality (9), we get

$$
(d-1) \cdot(n+r-2) \leq p \cdot(1-d / 2) \leq-p / 2 .
$$

If $p=0$ then $n+r \leq 2$ so $r+p \leq 2$ which is impossible since a meromorphic quadratic differential on $\mathbb{C P}^{1}$ has at least four poles. Thus $p>0$ and we again get $n+r \leq 1$.

The above remarks show that we can restrict our considerations to the following three (overlapping) cases:

$$
\left\{\begin{array} { l } 
{ n + r = 0 } \\
{ m = 0 }
\end{array} \quad \left\{\begin{array} { l } 
{ n + r = 1 } \\
{ m \geq 0 }
\end{array} \quad \left\{\begin{array}{l}
n+r=0 \\
m>0
\end{array}\right.\right.\right.
$$

To finish the proof of Lemma 3 we are going to show that all possible mappings with $d \geq 3$ satisfying (6) are listed in (2). We shall use the following obvious remark which is valid for any ramified covering $\pi: \tilde{M} \rightarrow M$ of degree $d$ and any point $P \in M$ :

$$
\sum_{\tilde{P} \in \pi^{-1}(P)} e_{\pi}(\tilde{P})=d
$$

The case $n+r=0$ and $m=0$. Since $n=r=m=0$ and genus $g=0$, we get a meromorphic quadratic differential with four simple poles $P_{1}, \ldots, P_{4}$ on $\mathbb{C P}^{1}, p=4$. Let $t_{i}$ be the number of points in the fiber $\pi^{-1}\left(P_{i}\right)(i=1, \ldots, 4)$ of ramification index two.

Taking the sum of expressions (10) over $P_{i}, i=1, \ldots, 4$, we get

$$
\begin{aligned}
4 d=\sum_{i=1}^{4} \sum_{\tilde{P} \in \pi^{-1}\left(P_{i}\right)} e_{\pi}(\tilde{P})=\sum_{i=1}^{4}\left(\sum_{\substack{\tilde{P} \in \pi^{-1}\left(P_{i}\right) \\
e_{\pi}(\tilde{P}) \neq 2}} e_{\pi}(\tilde{P})+\sum_{\substack{\tilde{P} \in \pi^{-1}\left(P_{i}\right) \\
e_{\pi}(\tilde{P})=2}} e_{\pi}(\tilde{P})\right) \\
=\sum_{i=1}^{4} \sum_{\substack{\tilde{P} \in \pi^{-1}\left(P_{i}\right) \\
e_{\pi}(\tilde{P}) \neq 2}} e_{\pi}(\tilde{P})+2 \sum_{i=1}^{4} t_{i} .
\end{aligned}
$$

On the other hand equation (6) gives

$$
(d-1) \cdot(4-2)=\sum_{i=1}^{4} t_{i}
$$

Note that, as $m=0$, the term corresponding to ramification index 1 in equation (6) 
does not appear. Thus we obtain

$$
\sum_{i=1}^{4} \sum_{\substack{\tilde{P} \in \pi^{-1}\left(P_{i}\right) \\ e_{\pi}(\tilde{P}) \neq 2}} e_{\pi}(\tilde{P})=4 .
$$

This means that either there are two ramification points $\tilde{P}_{1}, \tilde{P}_{2}$ of ramification index different from 2 , and then $e_{\pi}\left(\tilde{P}_{1}\right)=1, e_{\pi}\left(\tilde{P}_{2}\right)=3$, or there are four ramification points $\tilde{P}_{i}, i=1, \ldots, 4$ of ramification index different from 2 , and then $e_{\pi}\left(\tilde{P}_{i}\right)=1$.

The first solution suggests the map of the moduli spaces

$$
\mathcal{Q}(-1,-1,-1,-1) \longrightarrow \mathcal{Q}(-1,1) \text {. }
$$

But the stratum $\mathcal{Q}(-1,1)$ is empty (see above). The second solution corresponds to the map $\mathcal{Q}(-1,-1,-1,-1) \longrightarrow \mathcal{Q}(-1,-1,-1,-1)$ which is one of the maps $(2)$, and hence has no interest for us.

The case $n+r=1$ and $m \geq 0$. In this case either $n=1$ or $r=1$; denote by $k$ the order of corresponding singularity of $\psi$. The singularity is a true zero if $k>0$ or a pole if $k=-1$; by convention on notations $n, r$ it cannot be a marked point. With this notation $p=4+k$. We derive from (9) the following inequality: $-(d-1) \leq(4+k) \cdot(1-d / 2)-m$ which gives $d \leq 3-k / 2-m$. If $m \geq 1$, as $d \geq 3$, we obtain $k \leq-2$ which is forbidden since we consider only simple poles. Thus we must have $m=0$ and so $k=-1$. This implies that $n=0$ and $r=1$.

By inserting in inequality (9) we obtain $d=3$ or 4 . The stronger inequality (8) eliminates the solution $d=3$, so finally we get the solution $d=4$, where the values of the other parameters are as follows: $g=n=m=0 ; r=1$, and the corresponding point is a simple pole; there are three more simple poles, so $r=3$.

However, this solution gives the map $\mathcal{Q}(-1,-1,-1,-1) \rightarrow \mathcal{Q}(-1,-1,-1,-1)$ of the moduli spaces from the exceptional list (2), and hence has no interest for us.

The case $n+r=0$ and $m>0$. Since $n=r=0$ and genus $g=0$, we get a meromorphic quadratic differential with $p=4$ simple poles on $\mathbb{C P}^{1}$ and with $m>0$ marked points. Using inequality (8) we derive that if $d$ is odd, we have $m=0$ which is a contradiction. So $d$ must be even and again by using this inequality with $d$ even, we deduced that $1 \leq m \leq 2$.

If $m=1$, then we have a single marked point $P_{0} \in M$. We derive the following relation from equation (6):

$$
\begin{aligned}
& (d-1) \cdot(1+4-2) \\
& =\sum_{\text {poles } P_{i}} \text { number of preimage } \tilde{P} \text { in } \pi^{-1}\left(P_{i}\right) \text { of ramification index two } \\
& \quad+\text { number of preimage } \tilde{P} \in \pi^{-1}\left(P_{0}\right) \text { of ramification index one }
\end{aligned}
$$


Let $t$ be the number of preimage $\tilde{P} \in \pi^{-1}\left(P_{0}\right)$ of ramification index one over the marked point $P_{0}$. By Convention, at least one preimage of a marked point has ramification index greater then one, thus (10) implies $t \leq d-2$. Taking into consideration (7) we obtain from the equality above the following inequality

$$
3 \cdot(d-1) \leq 2 \cdot d+t
$$

and hence we have

$$
d-3 \leq t \leq d-2
$$

It is not difficult to see that if $t=d-3$ we obtain the following map

$$
\mathcal{Q}(-1,-1,-1,-1,0) \rightarrow \mathcal{Q}(4)
$$

but $\mathcal{Q}(4)$ is empty.

If $t=d-2$ the equality above implies that

$\sum_{P_{i} \text { poles }}$ number of preimages $\tilde{P}$ in $\pi^{-1}\left(P_{i}\right)$ of ramification index two $=2 \cdot d-1$ and taking in consideration (10) we get

$$
\sum_{i=1}^{4} \sum_{\substack{\tilde{P} \in \pi^{-1}\left(P_{i}\right) \\ e_{\pi}(\tilde{P}) \neq 2}} e_{\pi}(\tilde{P})=2
$$

where $P_{1}, \ldots, P_{4}$ are the simple poles. It is not difficult to see that in this case we obtain the map

$$
\mathcal{Q}(-1,-1,-1,-1,0) \rightarrow \mathcal{Q}(-1,-1,2)
$$

which belongs to the list (2) and hence does not interest us.

By similar arguments we conclude that if $m=2$ we get the map

$$
\mathcal{Q}(-1,-1,-1,-1,0,0) \rightarrow \mathcal{Q}(2,2)
$$

which belongs to the list (2) and hence does not interest us.

Lemma 3 is proved.

It remains to consider the two-fold coverings in order to complete the proof of Theorem 1.

Lemma 4. If $d=2$ then the strata obey the relation

$$
\operatorname{dim} \mathcal{Q}=\operatorname{dim} \tilde{\mathcal{Q}}
$$

only in the cases listed in Theorem 1 and in exceptional list (2).

Proof of Lemma 4. We suppose that the dimensions coincide. As the degree of the covering is two, there are no ramification points in $\tilde{M}$ of index one over 
marked points $P_{n+1}, \ldots, P_{n+m}$ on $M$, and there is exactly one point over each pole $P_{n+m+1}, \ldots, P_{n+m+r}$ in the set of critical values of $\pi$. Thus the equation (6) transforms into the following one

$$
\begin{gathered}
2 g+n+m+p+r-2=0+p=p, \\
2 g+n+m+r-2=0 .
\end{gathered}
$$

This equation shows that the genus $g$ of the underlying surface might be either 1 or 0 . If $g=1$ we get $n=m=r=0$ and hence the meromorphic quadratic differential on a surface of genus $g=1$ does not have either zeros nor marked points. Hence it does not have simple poles neither, which implies that it is the square of an Abelian differential.

Thus $g=0$ and $n+m+r=2$. We consider separately the following three cases:

$$
\left\{\begin{array} { l } 
{ n + m = 0 } \\
{ r = 2 }
\end{array} \quad \left\{\begin{array} { l } 
{ n + m = 1 } \\
{ r = 1 }
\end{array} \quad \left\{\begin{array}{l}
n+m=2 \\
r=0 .
\end{array}\right.\right.\right.
$$

The first two cases give us either the first two maps from Definition 1 or the exceptional map $\mathcal{Q}(-1,-1,-1,-1) \rightarrow \mathcal{Q}(-1,-1,-1,-1)$ from the list $(2)$.

The third case gives the mapping (when it is defined)

$$
\mathcal{Q}\left(k_{1}, k_{2},-1^{k_{1}+k_{2}+4}\right) \rightarrow \mathcal{Q}\left(2 k_{1}+2,2 k_{2}+2\right)
$$

with $k_{i} \geq 0$.

A ramified double covering over $\mathbb{C P}^{1}$ has even number of ramification points. Since in our case we have ramification points over the two singularities of degrees $k_{1}$ and $k_{2}$ and over all simple poles, this implies that the number $k_{1}+k_{2}+6$ is even, and hence $k_{1}$ and $k_{2}$ has the same parity. When both of them are odd, one can recognize in (11) the canonical ramified double covering described in Construction 1. Thus in this case the resulting quadratic differential is the global square of an Abelian differential, and this case does not interest us.

When both $k_{1}, k_{2} \geq 0$ are even we obtain the map of the third type from Definition 1. This completes the proof of Lemma 4.

Theorem 1 now follows immediately from Lemmas 3 and 4 .

\section{Quadratic differentials versa flat structures}

In this section we present a well-know relation between quadratic differentials and flat structures on Riemann surfaces. We use this relation to prove that the strata, that possess a hyperelliptic connected component, are not connected except several particular cases in low genera.

A flat surface with cone type singularities is a surface which possesses locally the geometry of a standard cone. We can define it by a flat Riemannian metric 
with specific isolated singularities. The standard cone possesses a unique invariant: it is the angle at the vertex. Here we consider only half-translation flat surfaces: parallel transport of a tangent vector along any closed path either brings the vector $\vec{v}$ back to itself or brings it to the centrally-symmetric vector $-\vec{v}$. This implies that the cone angle at any singularity of the metric is an integer multiple of $\pi$.

Let $\psi$ be a meromorphic quadratic differential on a Riemann surface $M_{g}^{2}$. Then it is possible to construct an atlas on $M \backslash$ \{singularities $\}$ such that $\psi=d z^{2}$ in any coordinate chart. As $d z^{2}=d w^{2}$ implies $z= \pm w+$ const, we see that the charts of the atlas are identified either by a translation or by a translation composed with a central symmetry. Thus a meromorphic quadratic differential $\psi$ induces a halftranslation flat structure on $M \backslash$ singularities $\}$. On a small chart which contains a singularity, coordinate $z$ can be chosen in such way that $\psi=z^{k} d z^{2}$, where $k$ is the order of the singularity ( $k=0$ corresponds to a regular point, $k=-1$ corresponds to a pole and $k>0$ corresponds to a true zero). It is easy to check that in a neighborhood of a singularity of $\psi$, the metric has a cone type singularity with the cone angle $(k+2) \pi$.

A meromorphic quadratic differential $\psi$ also defines on $M$ a pair of transversal foliations. Let $z$ be a canonical coordinate for $\psi$ i.e. let locally outside of singularity $\psi=(d z)^{2}$. The horizontal (respectively vertical) foliation defined by $\psi$ is the foliation $y=$ const (resp $x=$ const) locally where $z=x+i y$. The vertical (horizontal) foliation defined by $\psi$ is oriented if and only if the quadratic differential $\psi$ is the square of an Abelian differential.

Similarly, an Abelian differential defines a translation structure on the Riemann surface: now the holonomy representation of the corresponding flat metric in the linear group is trivial. In particular, all cone angles at the singularities are integer multiples of $2 \pi$.

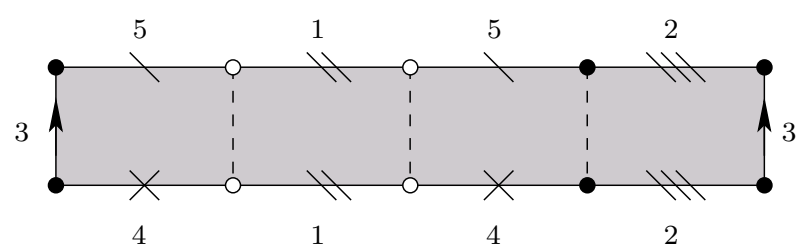

FIG. 3. Consider the following identifications of the boundary of this polygon: identify the corresponding pairs of segments with the numbers 1,2 and 3 by translations; identify the corresponding pairs of segments with the numbers 4 and 5 by centrally symmetries. We get a half-translation surface. In this case it has genus $g=2$ and the meromorphic differential induced from the quadratic differential $(d z)^{2}$ in the plane has two zeros of order 2 on this surface. The singularities are conical point in the flat metric with a cone of angle $4 \pi$. Note that since the horizontal foliation is not oriented the corresponding quadratic differential is not the square of an Abelian differential, though all cone angles of the singularities in the flat metric are integer multiples of $2 \pi$.

Reciprocally, a half-translation structure on a Riemann surface $M$ and a choice of a distinguished "vertical" direction defines a complex structure and a meromor- 
phic quadratic differential $\psi$ on $M$. In many cases it is very convenient to present a quadratic differential with some specific properties by an appropriate flat surface. Consider, for example, a polygon in the complex plane $\mathbb{C}$ with the following property of the boundary: the sides of the polygon are distributed into pairs, where the sides in each pair are parallel and have equal length. Identifying the corresponding sides of the boundary by translations and central symmetries we obtain a Riemann surface with a natural half-translation flat structure. The quadratic differential $d z^{2}$ on $\mathbb{C}$ gives a quadratic differential on this surface with punctures. The punctures correspond to vertices of the polygon; they produce the cone type singularities on the surface. It is easy to see that the complex structure, and the quadratic differential extends to these points, and that a singular point of the flat metric with a cone angle $(k+2) \pi$ produces a singularity of order $k$ (a pole if $k=-1$ ) of the quadratic differential. (See also Figure 3 which illustrates this construction.)

Remark 9. The area of $M$ with respect to the flat metric defined by a meromorphic quadratic differential is finite if and only if $\psi$ does not possess poles of order greater than 1 . This explains why we consider quadratic differential with simple poles only.

\subsection{Non-connectedness of the strata}

Now, we prove that strata with a hyperelliptic connected component are not connected in general. We present a particular geometric property of surfaces, that belong to a hyperelliptic component, and then we construct appropriate flat surfaces which do not verify this property.

Let us consider a flat surface $(M, \psi)$ in a hyperelliptic component of the stratum $\mathcal{Q}(4 k+2,4(g-k)-6)$, where $k \geq 0, g \geq 2, g-k \geq 2$. By definition, there exist a ramified double covering $\pi: M \rightarrow \mathbb{C P}^{1}$ such that $\psi=\pi^{*} \psi_{0}$ where $\psi_{0}$ is a meromorphic quadratic differential on $\mathbb{C P}^{1}$. We consider the canonical atlas on $M$ such that locally $\psi=d z^{2}$ in a neighborhood of a regular point and $\psi=z^{k} d z^{2}$ in a neighborhood of a singularity. In this atlas, the hyperelliptic involution $\tau: M \rightarrow$ $M$ is affine. Moreover, $\tau$ is an isometry of the flat metric defined by $\psi$. The two zeros of $\psi$ are fixed points of $\tau$.

Suppose that there exist a geodesic saddle connection joining the two zeros - a geodesic segment in the flat metric defined by $\psi$ having no singularities in its interior, and having the two zeros of $\psi$ as the endpoints. Then this saddle connection have an image by $\tau$ of the same length and it is also a saddle connection.

So if there is a horizontal saddle connection (which is a singular leaf of the horizontal foliation joining the two singularities) then there exists another one of the same length going in the same direction.

In particular, if a quadratic differential $\psi$ having two zeros of orders $4 k+2$ and 
$4(g-k)-6$ defines a horizontal foliation such that all horizontal saddle connections between the two zeros have different lengths, then $(M, \psi)$ does not belong to the hyperelliptic component of $\mathcal{Q}(4 k+2,4(g-k)-6)$. Since the hyperelliptic component of this stratum is nonempty, it would imply that the stratum is not connected.

The similar argument can be applied to the strata $\mathcal{Q}(2(g-k)-3,2(g-k)-$ $3,2 k+1,2 k+1)$ and $\mathcal{Q}(2(g-k)-3,2(g-k)-3,4 k+2)$.

We use this idea to construct appropriate flat surfaces such that the corresponding quadratic differentials do not belong to hyperelliptic components. We use the following two Lemmas which are particular cases of the corresponding Lemmas of Eskin, Masur and Zorich ([EMZ], [MaZo]); see also analogous Proposition 4.7(b) in the paper $[\mathrm{HuMa}]$ of Hubbard and Masur.

Lemma 5. Consider a surface in $\mathcal{Q}\left(k_{1}, \ldots, k_{n}\right)$. Choose $l_{1}, l_{2} \in\{-1,1,2,3,4, \ldots\}$, as follows

- if $k_{1}$ is odd, $l_{1}+l_{2}=k_{1}, l_{i}$ any.

- if $k_{1}$ is even, $l_{1}+l_{2}=k_{1}, l_{i}$ even.

For any $\psi_{0} \in \mathcal{Q}\left(k_{1}, k_{2}, \ldots, k_{n}\right)$ and for any sufficiently small $\varepsilon>0$ (depending on $\left.\psi_{0}\right)$ it is possible to construct a deformation $\psi \in \mathcal{Q}\left(l_{1}, l_{2}, k_{2}, \ldots, k_{n}\right)$ of $\psi_{0}$ such that the corresponding flat metric has a horizontal saddle connection of length $\varepsilon$ joining the singularities $P_{1}$ and $P_{2}$ of orders $l_{1}$ and $l_{2}$.

The deformation can be chosen to be local: the flat metric does not change outside of a small neighborhood of the zero of multiplicity $k_{1}$.

Lemma 6. Consider a surface in $\mathcal{Q}\left(k_{1}, \ldots, k_{n}\right)$. Let $k_{1}$ be odd and let $k_{1}=l_{1}+$ $l_{2}+l_{3}$, where $l_{i} \in\{-1,1,2,3,4, \ldots\}$ are also odd. For any $\psi_{0} \in \mathcal{Q}\left(k_{1}, k_{2}, \ldots, k_{n}\right)$ and for any sufficiently small $\varepsilon>0$ (depending on $\psi_{0}$ ) it is possible to construct a deformation $\psi \in \mathcal{Q}\left(l_{1}, l_{2}, l_{3}, k_{2}, \ldots, k_{n}\right)$ of $\psi_{0}$ such that the corresponding flat metric has two horizontal saddle connection of length $\varepsilon$ joining the singularities $P_{1}, P_{2}$ and $P_{2}, P_{3}$ of orders $l_{1}, l_{2}, l_{3}$ correspondingly.

The deformation can be chosen to be local: the flat metric does not change outside of a small neighborhood of the zero of multiplicity $k_{1}$.

Proof of Lemma 5. Let $P_{0} \in M$ be the singularity of $\psi_{0}$ of order $k_{1}$. Consider a small metric disk $D$ with the center in $P_{0}$ and of radius $R$ in $M$ in the flat metric on $M$ defined by the quadratic differential $\psi_{0}$. We choose $R$ to be small enough, so that $D$ does not contain other singularities of $\psi_{0}$.

In fact, $D$ is glued from $k_{1}+2$ Euclidean half-disks of radius $R$ where the corresponding radii of the half-disks are pairwise identified, see the left sides of Figures 4 and 5 . Note that the pictures are schematic: the angle of every sector is actually equal to $\pi$.

To make a local deformation of the Euclidean metric inside $D$ we reglue the radii borders of Euclidean half-disks in a different way. Figure 4 illustrates how to 
break a zero of even degree $k_{1}$ when even number $k_{1}+2$ of half-disks are adjacent to the vertex into two zeros of even degrees $l_{1}$ and $l_{2}$. Now there are $l_{1}+2$ and $l_{2}+2$ half-disks adjacent to the corresponding vertices. Note that the angles of all sectors are, actually, again equal to $\pi$. Figure 5 illustrates how to break a zero of odd degree $k_{1}$ into a zero of even degree $l_{1}$ and a zero of odd degree $l_{2}$.
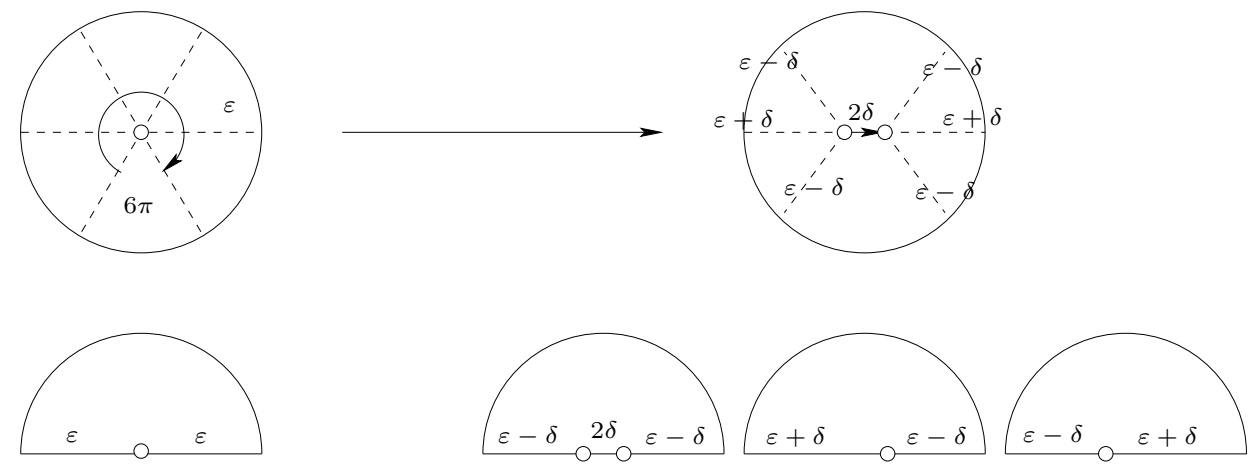

FIG. 4. Breaking up a zero of order 4 into two zeros of orders 2. Note that the surgery is local: we do not change the flat metric outside of the neighborhood of the zero.
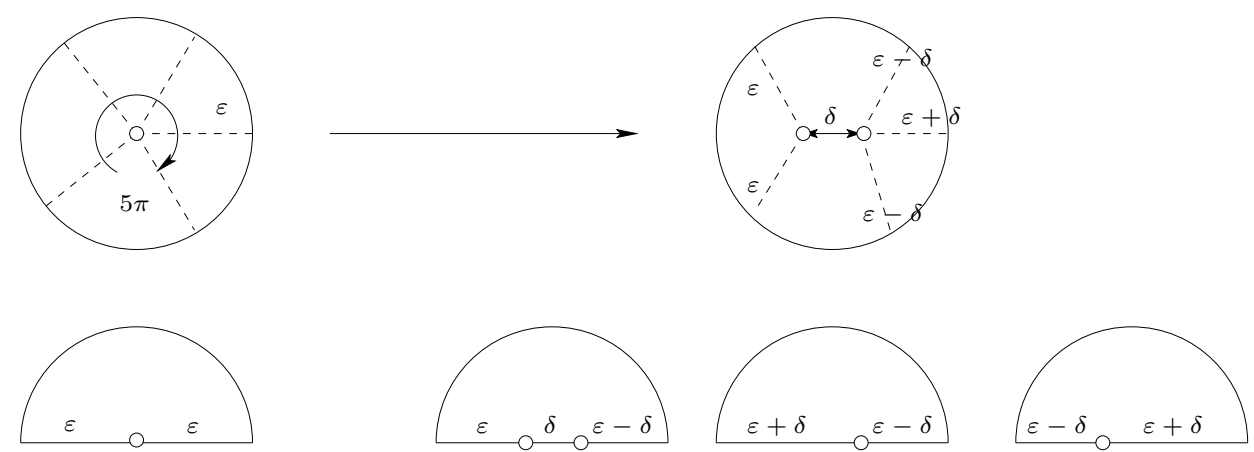

Fig. 5. Breaking up a zero of order 3 into two zeros of orders 1 and 2 correspondingly. Note that the surgery is local: we do not change the flat metric outside of the neighborhood of the zero.

Proof of Lemma 6 . The proof of Lemma 6 is completely analogous to the proof of Lemma 5. It is illustrated at figure 6. 

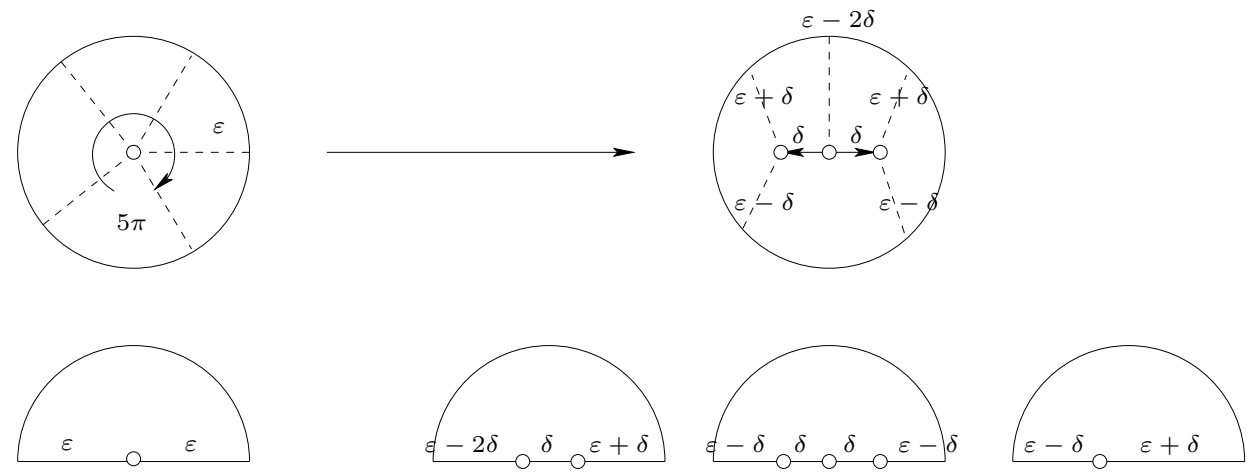

FIG. 6. Breaking up a zero of order 3 into three zeros of orders 1 . Note that the surgery is local: we do not change the flat metric outside of the neighborhood of the zero.

Theorem 2. The strata which possess a hyperelliptic component are non connected except for the following cases corresponding respectively to genus 1 and 2

$$
\left\{\begin{array} { l } 
{ \mathcal { Q } ( - 1 , - 1 , 2 ) } \\
{ \mathcal { Q } ( - 1 , - 1 , 1 , 1 ) }
\end{array} \quad \text { and } \left\{\begin{array}{l}
\mathcal{Q}(2,2) \\
\mathcal{Q}(1,1,2) \\
\mathcal{Q}(1,1,1,1)
\end{array}\right.\right.
$$

when the whole stratum coincides with its hyperelliptic connected component.

Proof of Theorem 2. We decompose the proof into three Lemmas. In the first, we prove that the strata of the list above are connected. In the second, we prove Theorem 2 for strata with 2 and 3 singularities. In the third we prove the Theorem for strata with fours singularities.

Lemma 7. The following strata are connected

$$
\begin{aligned}
& \mathcal{Q}(-1,-1,2), \mathcal{Q}(-1,-1,1,1) \text { in genus } 1, \\
& \mathcal{Q}(2,2), \mathcal{Q}(1,1,2), \mathcal{Q}(1,1,1,1) \text { in genus } 2,
\end{aligned}
$$

they coincide with the corresponding hyperelliptic component.

Proof of Lemma \%. We use an idea drawn from the book of Farkas-Kra [FaKr]. See also [La2] for another proof, using the combinatorics of "generalized permutations".

We prove this Lemma for $\mathcal{Q}(2,2)$. For the other strata, the demonstration is similar. First of all, take a point $[M, \psi] \in \mathcal{Q}(2,2)$. In genus 2, all curves are hyperelliptic so we can suppose that a representative of $M$ is given by an algebraic curve

$$
w^{2}=\prod_{i=1}^{6}\left(z-z_{i}\right) \quad(w, z) \in \mathbb{C}^{2}
$$


where $z_{i} \neq z_{j}$ for $i \neq j$ and $z_{i} \in \mathbb{C}$. There is a (ramified) double covering $\pi: M \rightarrow \mathbb{C P}^{1}$ defined by $\pi(w, z)=z$. Let $\tau$ be the hyperelliptic involution $\tau(w, z)=(-w, z)$. With these notations, we can see that $\omega_{1}=\frac{d z}{w}$ and $\omega_{2}=$ $z \cdot \frac{d z}{w}$ form a basis of holomorphic Abelian differentials on $M$. It easy to see that $\omega_{1}^{2}, \omega_{2}^{2}$ and $\omega_{1} \cdot \omega_{2}$ are linearly independent in the space of holomorphic quadratic differentials which has dimension $3 g-3=3$. So the family $\left\{\omega_{1}^{2}, \omega_{2}^{2}, \omega_{1} \cdot \omega_{2}\right\}$ forms a basis in the space of holomorphic quadratic differentials on $M$. By direct computation, we can see that $\tau^{*} \omega_{i}=-\omega_{i}$ and consequently, any holomorphic quadratic differential is invariant under $\tau$.

Let $(M, \psi) \in \mathcal{Q}(2,2)$ and let $P_{1}$ and $P_{2}$ be the two zeros of $\psi$. We have

$$
0=\psi\left(P_{1}\right)=\tau^{*} \psi\left(P_{1}\right)=\psi\left(\tau\left(P_{1}\right)\right)
$$

hence $\tau$ either fixes the two zeros or interchanges them.

Suppose that the second case occurs. Let $z_{0}=\pi\left(P_{1}\right)=\pi\left(P_{2}\right)$. Since by assumption $\tau\left(P_{1}\right) \neq P_{2}$, we see that $z_{0}$ is a noncritical value of the covering, and hence $z_{0} \neq z_{i}$ for any $i=1, \ldots, 6$. We can construct a quadratic differential $\psi_{0}$ on $\mathbb{C P}^{1}$ with six simple poles at the images $z_{i} \in \mathbb{C P}^{1}$ of the ramifications points, and a zero of order 2 at $z_{0}$. Then the covering $\pi: M \rightarrow \mathbb{C P} P^{1}$ is exactly the covering of Construction 1 so $\pi^{*} \psi_{0}=\omega^{2}$ possesses two zeros of order two at the same points as the two zeros of $\psi$. Hence $\psi=$ const $\cdot \omega^{2}$ which contradicts the assumption that $\psi$ is not a global square of an Abelian differential.

Thus the two zeros $P_{1}$ and $P_{2}$ are the fixed points of the hyperelliptic involution. Let $z_{0}=\pi\left(P_{1}\right), z_{0}^{\prime}=\pi\left(P_{2}\right)$. We can construct a quadratic differential $\psi_{0}$ on $\mathbb{C P}^{1}$ with four poles at the images of ramifications points, and with two fake zeros at $z_{0}$ and $z_{0}^{\prime}$ correspondingly. As above, $\psi=$ const $\cdot \pi^{*} \psi_{0}$. But by construction $\pi^{*} \psi_{0}$ belongs to the hyperelliptic connected component (see Construction 2). Thus $p \in \mathcal{Q}^{\text {hyp }}(2,2)$ and hence $\mathcal{Q}(2,2)=\mathcal{Q}^{\text {hyp }}(2,2)$ is connected.

This completes the proof of Lemma 7 in the case of the stratum $\mathcal{Q}(2,2)$. The proof for other cases is completely analogous.

Now we prove Theorem 2 for the general strata with 2 and 3 singularities.

Lemma 8. The strata

$$
\mathcal{Q}(4(g-k)-6,4 k+2) \text { with } k \geq 0, g \geq 3 \text { and } g-k \geq 2
$$

and

$$
\begin{gathered}
\qquad \mathcal{Q}(2(g-k)-3,2(g-k)-3,4 k+2) \\
\text { with } g \geq 3, k \geq 0, g-k \geq 1 \text { or } g=2 \text { and } k=1
\end{gathered}
$$

are non-connected. 
Proof of Lemma 8. By the Theorem of Masur and Smillie [MaSm] (see also the statement of the theorem at the beginning of section 2) the stratum $\mathcal{Q}(4 g-4)$ is non-empty when genus $g \geq 3$. Consider a flat surface corresponding to some $\left[M, \psi_{0}\right] \in \mathcal{Q}(4 g-4)$ such that the horizontal measured foliation is uniquely ergodic, in particular minimal. (The genericity of this property is proved in $[\mathrm{Ma}]$ and in [Ve1].) Consider a small disk around the zero of the quadratic differential. Applying Lemma above, we can break the zero of order $4 g-4$ into two zeros of orders $4(g-k)-6$ and $4 k+2$ correspondingly with a very short horizontal saddle connection $\gamma$ joining them. By construction there are no other short horizontal saddle connections. So the quadratic differential $\psi \in \mathcal{Q}(4(g-k)-6,4 k+2)$ thus constructed does not belong to hyperelliptic connected component of $\mathcal{Q}(4(g-k)-$ $6,4 k+2)$ (see the beginning of this section). This proves the first statement.

Consider now the stratum $\mathcal{Q}(2(g-k)-3,2(g+k)-1)$ with $k \geq 0, g-k \geq 1$. By the Theorem of Masur and Smillie [MaSm] this stratum is non-empty if $g \geq 3$ or if $g=2$ and $k=1$.

Take a flat surface corresponding to a quadratic differential from this stratum such that the corresponding horizontal foliation is minimal (see above). Then if we break the zero of order $2(g+k)-1$ into two zeros of orders $2(g-k)-3$ and $4 k+2$ joined by a very short horizontal saddle connection we get a surface which belongs to the non-hyperelliptic component of the stratum $\mathcal{Q}(2(g-k)-3,2(g-k)-3,4 k+2)$.

The only strata to which we cannot apply this method are the stratum with two poles and a simple zero $\mathcal{Q}(-1,-1,2)$ in genus 1 and the stratum with two simples zeros and a double zero $\mathcal{Q}(1,1,2)$ in genus 2 . In these two cases the initial (nonperturbed) quadratic differentials would belong to $\mathcal{Q}(-1,1)$ and $\mathcal{Q}(1,3)$ which are empty.

Lemma 8 is proved.

Let us prove proposition for strata with four singularities.

Lemma 9. The strata

$$
\begin{gathered}
\qquad \mathcal{Q}(2(g-k)-3,2(g-k)-3,2 k+1,2 k+1) \\
\text { with } g \geq 3, k \geq-1, g-k \geq 2 \text { or } g=2 \text { and } k=-1
\end{gathered}
$$

are non-connected.

Proof of Lemma 9. Consider the stratum $\mathcal{Q}(2(g-k)-3,2(g+k)-1)$ with $g \geq 3$, $k \geq-1, g-k \geq 2$. By the Theorem of Masur and Smillie [MaSm] this stratum is non-empty.

Take a flat surface corresponding to a quadratic differential from this stratum such that the corresponding horizontal foliation is minimal (see above). By Lemma 6 we can break the zero of order $2(g+k)-1$ into three zeros of orders $2(g-k)-3,2 k+1,2 k+1$, joined consecutively by very short horizontal saddle connections. We use a local perturbation of the flat metric, which not rather cre- 
ate a short horizontal saddle connection going to the unperturbed zero of order $2(g-k)-3$. We get a flat surface corresponding to a quadratic differential from the stratum $\mathcal{Q}(2(g-k)-3,2(g-k)-3,2 k+1,2 k+1)$.

The hyperelliptic involution interchanges the corresponding zeros of any $\psi^{\prime} \in$ $\mathcal{Q}^{h y p}(2(g-k)-3,2(g-k)-3,2 k+1,2 k+1)$. By construction the quadratic differential constructed above is asymmetric, and hence it belongs to the nonhyperelliptic component of the stratum $\mathcal{Q}(2(g-k)-3,2(g-k)-3,4 k+2)$. Lemma 9 is proved.

Now, Theorem 2 follows from Lemmas 8 and 9 .

\section{Announcement of the Classification Theorem}

In [KoZo] Kontsevich and Zorich have shown that the connected components of the moduli spaces of Abelian differentials are classified exactly by two invariants: the hyperellipticity and the parity of the spin structure.

The story for the moduli space of quadratic differential is more complicated. For example, the strata $\mathcal{Q}(12)$ and $\mathcal{Q}(-1,9)$ do not have any hyperelliptic components. In addition, in paper [La1], we show that all quadratic differentials in a fixed strata must have the same parity of the spin structure. In the previous case, the spin structure is 0 for any quadratic differential in each of these two strata. However, it was proved by A. Zorich by a direct computation of the corresponding extended Rauzy classes that each of these two strata has exactly two distinct connected components.

In [La2] we give the following general description of all connected components of any stratum of the moduli spaces $\mathcal{Q}\left(k_{1}, \ldots, k_{n}\right)$ :

Theorem 3. Let us fix $g \geq 5$. Then all strata of the moduli space $\mathcal{Q}_{g}$ listed in Theorem 2 have exactly two connected components: one is hyperelliptic - the other not.

All other strata of the moduli space of meromorphic quadratic differentials $Q_{g}$ are non-empty and connected.

And for small genera we have:

Theorem 4. Let us fix $g \leq 4$. The components of the strata of the moduli space $\mathcal{Q}_{g}$ fall in the following description

- In genera 0 and 1 , any stratum is connected.

- In genus 2 there are two hyperelliptic non-connected strata. All other strata of $\mathcal{Q}_{2}$ are connected.

- In genera 3 and 4, any hyperelliptic stratum possesses two connected components: one is hyperelliptic - the other not. All other strata, with 4 exceptional cases, are connected. 
- The 4 above particular cases are

$$
\mathcal{Q}_{g=3}(-1,9), \mathcal{Q}_{g=3}(-1,3,6), \mathcal{Q}_{g=3}(-1,3,3,3), \mathcal{Q}_{g=4}(12)
$$

and these strata have two connected components.

Acknowledgments. I would like to thank A. Zorich for his proposition to work with such beautiful objects like quadratic differentials and moduli spaces. I am grateful to A. Zorich, P. Hubert and the Referee for their remarks concerning this paper.

\section{References}

[A] M. Atiyah, Riemann surfaces and spin structures, Ann. scient. Ec. Norm. Sup. 4 (1971), 47-62.

[EMZ] A. Eskin, H. Masur and A. Zorich, Moduli spaces of Abelian differentials: the principal boundary, counting problems and the Siegel Veech constants, Publ. Math. Inst. Hautes Études Sci. 97 (2003), 61-179.

[EsOk] A. Eskin and A. Okounkov, Asymptotics of number of branched coverings of a torus and volumes of moduli spaces of holomorphic differentials, Invent. Math. 145:1 (2001), 59-104.

[FaLaPo] A. Fathi, F. Laudenbach et V. Poenaru, Travaux de Thurston sur les surfaces, Astérisque 66-67 (1979).

[FaKr] H. Karkas and I. Kra, Riemann surfaces, Second edition, Graduate Texts in Mathematics 71, Springer-Verlag, New York, 1992.

[HuMa] J. Hubbard and H. Masur: Quadratic differentials and foliations, Acta Math. 142 (1979), 221-274.

[Ko] M. Kontsevich, Lyapunov exponents and Hodge theory, in: The mathematical beauty of physics (Saclay, 1996, in Honor of C. Itzykson), Adv. Ser. Math. Phys. 24, 318-332 World Sci. Publishing, River Edge, NJ, 1997.

[KoZo] M. Kontsevich and A. Zorich, Connected components of the moduli spaces of Abelian differentials with prescribed singularities, Invent. Math. 153 no. 3 (2003), 631-678.

[Jo] D. Johnson, Spin structures and quadratic forms on surfaces, J. London Math. Soc. 22 (1980), 365-373.

[La1] E. Lanneau, Parity of the Spin Structure Defined by a Quadratic Differential, Geometry Es topology $8(2004), \mathrm{n}^{\mathrm{O}} 12,511-539$.

[La2] E. Lanneau, Classification of connected components of the strata of the moduli spaces of quadratic differentials with prescribed singularities, Thèse de Doctorat, Université de Rennes 1, Décembre 2003.

[Ma] H. Masur, Interval exchange transformations and measured foliations, Ann. of Math. 115:1 (1982), 169-200.

[MaSm] H. Masur and J. Smillie, Quadratic differentials with prescribed singularities and pseudo-Anosov diffeomorphisms, Comment. Math. Helvetici 68, 289-307 (1993).

[MaTa] H. Masur and S. Tabachnikov, Flat structures and rational billiards, in: Handbook of dynamical systems, Vol. 1A, 1015-1089, North-Holland, Amsterdam, 2002.

[MaZo] H. Masur and A. Zorich, The principal boundary of the moduli spaces of quadratic differentials, Publ. Math. Inst. Hautes Études Sci. 97 (2003), 61-179.

[Mi] J. Milnor, Remarks concerning spin manifolds, Differential and Combinatorial Topology (in Honor of Marston Morse) Princeton, 1965.

[St] K. Strebel, Quadratic differentials, Springer-Verlag, 1984. 
[Ve1] W. A. Veech, Gauss measures for transformations on the space of interval exchange maps, Annals of Mathematics 115 (1982), 201-242.

[Ve2] W. A. Veech, Moduli spaces of quadratic differentials, Journal d'Analyse Math. 55 (1990), 117-171.

Erwan Lanneau

Institut de Mathématiques de Luminy (IML)

163 Avenue de Luminy

Case 907

13288 Marseille Cedex 9

France

e-mail: lanneau@iml.univ-mrs.fr

(Received: June 10, 2002)

(D) To access this journal online:

(4) http://www.birkhauser.ch 